\title{
Particle-in-Cell Simulations of Relativistic Weibel-Mediated Shocks Propagating into Inhomogeneous Media
}

\author{
Sara Tomita* \\ Department of Physics and Mathematics, Aoyama Gakuin University 5-10-1 Fuchinobe, \\ Sagamihara 252-5258, Japan \\ E-mail: tomisaradphys.aoyama.ac.jp

\section{Yutaka Ohira} \\ Department of Earth and Planetary Science, The University of Tokyo 7-3-1 Hongo, Bunkyo-ku, \\ Tokyo 113-0033, Japan \\ E-mail: \&.ohiradeps.s.u-tokyo.ac.jp
}

\begin{abstract}
The cosmic rays above the second knee energy $\left(10^{17} \mathrm{eV}\right)$ are expected to be accelerated by extragalactic sources, such as relativistic jets of gamma-ray bursts (GRBs) and active galactic nucleus. The relativistic jets drive a relativistic Weibel mediated shock if the upstream magnetic field is sufficiently weak. External shocks of GRBs can accelerate CRs to $10^{17} \mathrm{eV}$ if the magnetic field generated by the Weibel instability around the shock front do not decay in the far downstream region. However, particle-in-cell (PIC) simulations show that the magnetic fields decay rapidly for the Weibel mediated shocks in a uniform plasma. In reality, there are density fluctuations in interstellar or circumstellar medium. In this study, we preformed two-dimensional PIC simulations of relativistic shocks propagating to inhomogeneous electron-positron plasma. We found that the downstream magnetic field keeps a higher values and larger scales than those for the uniform case. Furthermore, sound and entropy waves are excited by interaction of the upstream density fluctuations with the shock, which would accelerate particles to a higher energy by the second-order acceleration. Therefore, the upstream inhomogeneity plays a crucial role in the CR acceleration and generation of the magnetic field in the far downstream region.
\end{abstract}

36th International Cosmic Ray Conference -ICRC2019-

July 24th - August 1st, 2019

Madison, WI, U.S.A.

${ }^{*}$ Speaker. 


\section{Introduction}

The energy range of cosmic rays is very wide, starting from $10^{8} \mathrm{eV}$ and reaching about $10^{20}$ $\mathrm{eV}$. The origins of CRs less than $10^{17} \mathrm{eV}$ are believed to be galactic sources, such as supernova remnants and pulsar wind nebulae. On the other hand, CRs above $10^{17} \mathrm{eV}$ are expected to be accelerated by extragalactic sources, such as relativistic jets of gamma-ray bursts (GRBs) and active galactic nucleus. The relativistic jet interacts with the interstellar medium (ISM) or circumstellar medium(CSM), where the bulk flow of plasma is dissipated and heated by the magnetic field generated by the Weibel instability. The Weibel instability is driven by anisotropic-temperature plasmas [2]], which is thought to be important for the generation of magnetic fields and particle

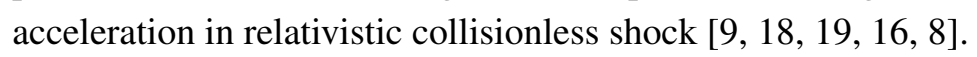

The relativistic Weibel mediated shock propagating to a uniform plasma has been investigated by many Particle-in-cell (PIC) simulations [B], 四]. It is shown that the magnetic field generated by the Weibel instability around the shock front rapidly decays. In addition, the small-scale magnetic fluctuation makes the particle acceleration inefficient. Even if the generated magnetic field does not decay in the downstream region, the expected maximum energy of CR protons becomes about $10^{17} \mathrm{eV}$ for GRB afterglows, which cannot explain the extragalactic high-energy CRs.

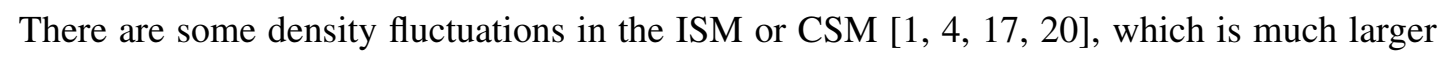
than the kinetic scale. In addition, smaller density fluctuations are also produced in a precursor region of collisionless shocks propagating into homogeneous media [15, [2], [13, ㄴ4]]. In magnetohydrodynamics (MHD) simulations, it is found that the downstream magnetic field is amplified by the turbulent dynamo when the shock propagates into inhomogeneous media [ [

Since the MHD simulation cannot solve any physics in the kinetic scale, in this study, by performing PIC simulations, we investigate the relativistic collisionless shocks propagating into inhomogeneous media. We found that, the downstream magnetic field keeps a higher amplitude and larger scale than that for the uniform case. Furthermore, sound and entropy waves are excited in the shock downstream region by an interaction of incident waves with shock waves. We could expect that the upstream inhomogeneity is crucial role for the generation of magnetic field and particle acceleration in relativistic collisionless shocks.

\section{Simulation}

\subsection{Setting}

We use the two-dimensional electromagnetic PIC code, pCANS [15]]. The two-dimensional simulation box is in the $x-y$ plane where the periodic boundary condition is imposed in the $y-$ direction. The simulation box size is $L_{x}=1.2 \times 10^{4} c / \omega_{\mathrm{pe}}$, and $L_{y}=86.4 \times c / \omega_{\mathrm{pe}}$. The plasma skin-depth of the upstream electron-positron plasma, $c / \omega_{\text {pe }}$, is given by

$$
\frac{c}{\omega_{\mathrm{pe}}}=\sqrt{\frac{\Gamma m_{\mathrm{e}} c^{2}}{4 \pi n_{0} e^{2}},}
$$

where $n_{0}$ and $\Gamma$ are the upstream mean number density and bulk Lorentz factor, and the constants $c, e, m_{\mathrm{e}}$ are speed of light, particle charge, particle mass, respectively. The cell size and time step are 
$\Delta x=\Delta y=0.1 c / \omega_{\mathrm{pe}}$ and $\Delta t=0.1 \omega_{\mathrm{pe}}^{-1}$, respectively. Incoming unmagnetized plasma with Lorentz factor $\gamma=10$ and thermal velocity $v_{\text {th }}=0.1 c$ is reflected at the right boundary $\left(x=1.2 \times 10^{4} c / \omega_{\text {pe }}\right)$. We use 40 particles per cell per species. The initial spatial distribution of the electron-positron plasma in the upstream region is set to be

$$
n(x, y)=n_{0}\left[1+0.5 \sin \left(2 \pi x / \lambda_{x}\right)\right]
$$

Namely, the density fluctuation is given by a monochromatic wave with $\delta n / n_{0}=0.5$. The wavelength is set to be $1.2 \times 10^{3} \mathrm{c} / \omega_{\text {pe }}$.

\subsection{Result}

Figure 1 shows the shock structure for the case of homogeneous and inhomogeneous density distributions. The shock font is located at $x=x_{\mathrm{sh}}$. The left and right sides are the upstream and downstream regions, respectively. The panels (a) and (b) in Figure $\mathbb{W}$ show the shock structure for the homogeneous case at $t \omega_{\mathrm{pe}}=5000$. The two panels show the two-dimensional structures of electron-positron density, the energy fraction of the magnetic field $\varepsilon_{B}=B^{2} /\left(32 \pi \Gamma n_{0} m_{e} c^{2}\right)$. For the uniform case, the Weibel filaments are generated in the shock transition region and the magnetic field simply decays in the downstream region. The panels (c)-(f) in Figure $\mathbb{W}$ show the shock structure when the shock front is passing through the highest- and lowest-density regions, respectively. The panels (c) and (d) show that there are large-scale filaments in the downstream region and the magnetic-field strength is comparable to that in the shock front. In the panels (e) and (f), the transverse width of the upstream filaments is significantly larger than that for the homogeneous case. In addition, the magnetic field is generated in the broad upstream region. The large-scale magnetic field can be advected far downstream without dissipation because the dissipation time becomes longer.

Figure $\square$ shows the spatial profiles of the transversely averaged $\varepsilon_{\mathrm{B}}$ in the larger downstream region at $t \omega_{\mathrm{pe}}=6100$, where the panel (a) and (b) show $\varepsilon_{\mathrm{B}}$ for homogeneous and inhomogeneous cases, respectively. The magnetic field simply decays near the shock front for the homogeneous case. On the other hand, for inhomogeneous case, there are two peaks of $\varepsilon_{\mathrm{B}}$ in the downstream region. The number of the peaks is same as that of the waves which transmitted through the shock. The magnetic field in the peaks was generated when the shock propagated in the low-density regions, converted toward downstream.

Finally, we show spatial profiles of the density and velocity in large downstream region for inhomogeneous case. The blue and red curves in Figure 3 show the one-dimensional structures of $x$ component of the average velocity, $\left\langle v_{x}\right\rangle / c$, and density, $n / n_{0}$, at $t \omega_{\mathrm{pe}}=6100$. We can see that the velocity profile almost correlates with the large-scale density fluctuation. Thus, the sinusoidal variation can be interpreted as a sound wave propagating downstream. As shown in red curve in Figure B, we can see another intermediate-scale density fluctuation with a wavelength of about 400 $c / \omega_{\mathrm{pe}}$ at $x-x_{\mathrm{sh}} \approx 350,850,1250,1650 c / \omega_{\mathrm{pe}}$. They are not associated with the velocity variation and do not propagate. Therefore, we can interpret this intermediate-scale density fluctuation as an entropy wave. The observed wavelengths of the entropy and sound waves are consistent with results from hydrodynamic analysis of interaction between the shock front and the density fluctuation. 


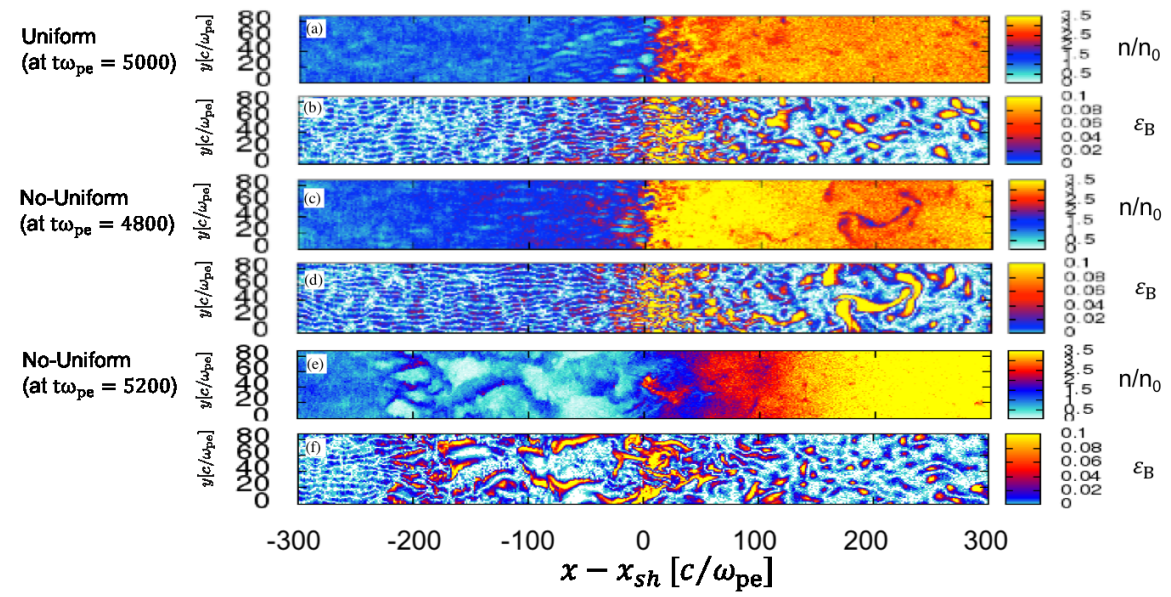

Figure 1: Structures of shocks for the homogeneous density distribution at $t \omega_{\mathrm{pe}}=5000$ (top panels), and inhomogeneous case at $t \omega_{\mathrm{pe}}=4800$ (middle panels) and 5200 (bottom panels). At $t \omega_{\mathrm{pe}}=4800$ and 5200 , the shock front is passing through the highest- and lowest-density regions, respectively. Here, the $x-x_{\mathrm{sh}}$ is the distance from the shock which is located at $x_{\mathrm{sh}}$. Panels (a), (c), and (e) show the plasma density normalized by the upstream mean plasma density, $n / n_{0}$. Panels (b), (d), and (f) show the magnetic energy density normalized by the upstream mean kinetic energy density, $\varepsilon_{\mathrm{B}}=B^{2} / 32 \pi \Gamma n_{0} m_{\mathrm{e}} c^{2}$.
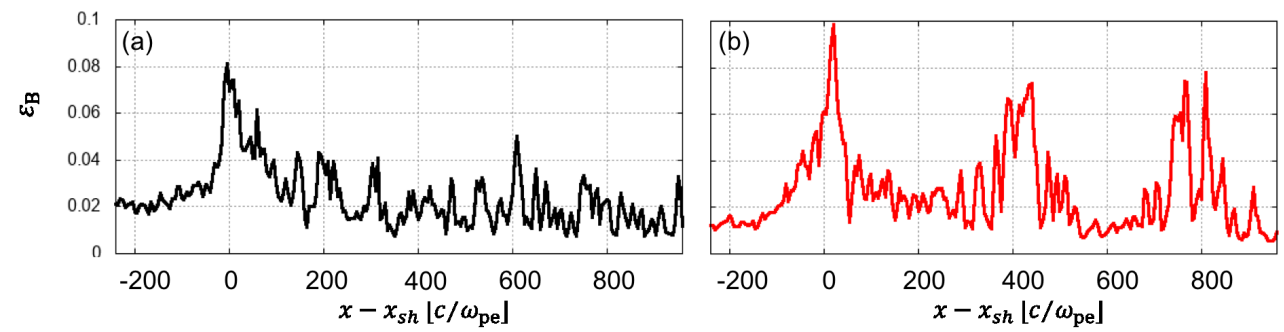

Figure 2: Spatial profiles of the transversely averaged $\varepsilon_{\mathrm{B}}=B^{2} / 32 \pi \Gamma n_{0} m_{\mathrm{e}} c^{2}$ in the larger downstream region at $t \omega_{\mathrm{pe}}=6100$ for the homogeneous (a) and inhomogeneous (b) density distributions, respectively. Here, the $x-x_{\mathrm{sh}}$ is the distance from the shock which is located at $x_{\mathrm{sh}}$.

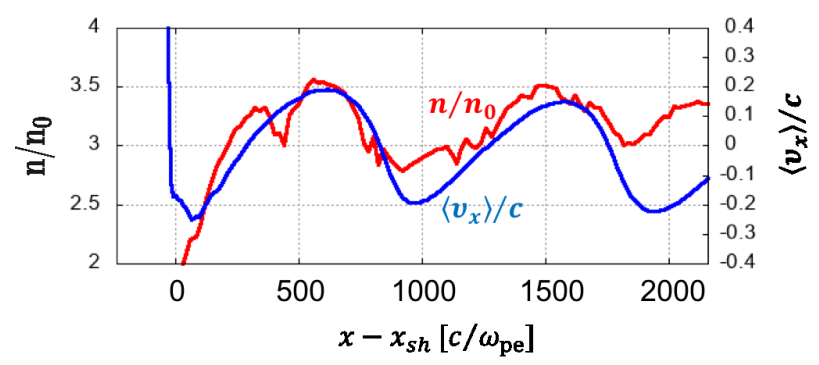

Figure 3: Spatial profiles of the $x$ component of the average velocity, $\left\langle v_{x}\right\rangle / c$ (blue), and density, $n / n_{0}$ (red), measured in the downstream rest frame at $t \omega_{\mathrm{pe}}=6100$ in the downstream region for the inhomogeneous upstream case. Here, the $x-x_{\mathrm{sh}}$ is the distance from the shock which is located at $x_{\mathrm{sh}}$. 


\section{Discussion}

We performed the PIC simulation of unmagnetized relativistic collisionless shock propagating into an inhomogeneous medium. Our simulation showed that the larger-scale magnetic field is generated by the Weibel instability in the shock precursor region when the shock is passing through the low-density region. The large-scale magnetic field can persist in the far downstream region without damping. We could expect that the particle acceleration becomes more efficiently in the relativistic Weibel-mediated shock for inhomogeneous upstream density because the larger-scale of the magnetic field fluctuation makes the particle scattering efficient. Moreover, our simulation showed that sound and entropy waves are generated in the downstream region of collisionless shock as predicted by the hydrodynamic analysis of the interaction between a shock and an incident density fluctuation. The sound wave could accelerate particles in the downstream region by the second order acceleration or the turbulent acceleration [ㅁ], [1]. The finite amplitude sound wave would eventually become shock like structures, so that the diffusive shock acceleration by the low Mach number shock might work in the shock downstream region. To confirm such particle acceleration in the downstream region, we need the larger and longer simulation.

\section{Acknowledgments}

We thank M. Hoshino, Y. Matsumoto, R. Yamazaki, T. Amano for useful comments. The software used in this work was in part developed in pCANS at Chiba University. Numerical computations were carried out on Cray X50 at Center for Computational Astrophysics, National Astronomical Observatory of Japan. This work is supported by Grant-in-Aid for JSPS Research Fellow from Japan Society for the Promotion of Science (JSPS), No. 17J03893 (ST), and in part by JSPS KAKENHI grant numbers 16K17702 (YO) and 19H01893 (YO).

\section{References}

[1] W., Armstrong, J., Rickett, \& S. R., Spangler, Electron density power spectrum in the local interstellar medium, ApJ, 443, 209, 1995.

[2] K., Asano, \& T., Terasawa, Stochastic acceleration model of gamma-ray burst with decaying turbulence, MNRAS, 454, 2242, 2015.

[3] P., Chang, A., Spitkovsky, \& J., Arons, Long-term evolution of magnetic turbulence in relativistic collisionless shocks: electron-positron plasmas, ApJ, 674, 378, 2008.

[4] N., Chugai, \& I., Danziger, SN 1988Z: low-mass ejecta colliding with the clumpy wind?, MNRAS, 268, 173, 1994.

[5] O., Drury, \& S., Falle, On the Stability of Shocks Modified by Particle Acceleration, MNRAS, 223, 353, 1986.

[6] T., Inoue, K., Asano, \& K., Ioka, Three-dimentinal simulations of magnetohydrodynamic turbulence behind relativistic shock waves and their implications for gamma-ray bursts, ApJ, 734, 77, 2011.

[7] T., Keshet, B., Katz, A., Spitkovsky, \& E., Waxman, Magnetic field evolution in relativistic unmagnetized collisonless shocks, ApJL, 693, L127, 2009. 
[8] Y., Matsumoto, T., Amano, T. N., Kato, \& M., Hoshino, Electron surfing and drift accelerations in a Weibel-dominated high-Mach-number shock, PRL, 119, $105101,2017$.

[9] M., Medvedev \& A., Loeb, Generation of magnetic fields in the relativistic shock of gamma-ray burst sources, ApJ, 526, 697-706, 1999.

[10] Y., Mizuno, M., Pohl, J., Niemiec, et al., Magnetic field amplification and saturation in turbulence behind a relativistic shock, ApJ, 726, 62, 2011.

[11] Y., Ohira, Turbulent shear acceleration, ApJ, 767, L16, 2013.

[12] Y., Ohira, Simulations of collisionless perpendicular shocks in partially ionized plasmas, PRL, 111, 245002, 2013.

[13] Y., Ohira, Acoustic instability in the neutral precursor region of collisionless shocks propagating into partially ionized plasmas, MNRAS, 440, 514, 2014.

[14] Y., Ohira, Magnetic field amplification by collisionless shocks in partially ionized plasmas, ApJ, 817, 137, 2016.

[15] pCANS, http://www.astro.phys.s.chiba-u.ac.jp/pcans/

[16] L., Sironi, A., Spitokovsky, \& J., Arons, The maximum energy of accelerated particles in relativistic collisionless shocks, ApJ, 771, 54, 2013.

[17] N., Smith, J., Silverman, R., Chornock, et al., Coronal lines and dust formation in SN 2005ip: not the brightest, but the hottest, ApJ, 695,1334, 2009.

[18] A., Spitokovsky, On the structure of relativistic collisionless shocks in electron-Ion plasmas, ApJ, 673, L39, 2008.

[19] A., Spitkovsky, Particle acceleration in relativistic collisionless shocks: Fermi process at last?, ApJ, 682, L5-L9, 2008.

[20] A., Yalinewich, \& P. S., Zwart, The Signature of a Windy Radio Supernova Progenitor in a Binary System, ApJL, 872, L3, 2019.

[21] E. S., Weibel, Spontaneously growing transverse waves in a plasma due to an anisotropic velocity distribution, PRL, 2, 83-84, 1959. 